\title{
First intraindividual comparison of contrast- enhanced MRI, FET- and DOTATOC- PET in patients with intracranial meningiomas
}

\author{
Jan Oliver Dittmar ${ }^{1,2}$, Clemens Kratochwil $^{3}$, Anne Dittmar ${ }^{2}$, Thomas Welzel ${ }^{2}$, Daniel Habermehl ${ }^{1,4,5}$, Stefan Rieken²,
} Frederik L. Giesel ${ }^{3}$, Uwe Haberkorn ${ }^{3}$, Jürgen Debus ${ }^{2}$ and Stephanie E. Combs ${ }^{1 *}$

\begin{abstract}
Background: For irradiation treatment planning of meningiomas the use of PET-scans is well established. The most frequently used tracers are either based on amino acids or the somatostatin receptor ligand DOTATOC. Since up to now no inter-institutionally accepted standard PET-tracer has been defined, the aim of this study was to evaluate the influence of these different types of PET-tracers on the GTV-definition.

Methods: Twenty-one patients suffering from intracranial meningiomas underwent CT, MRI, FET- and DOTATOC-PET. First, tumour extension was delineated after image-fusion of $C T$ and MRI (GTV $\left.\mathrm{CTTRR}_{\mathrm{MR}}\right)$. Then distinct GTVs based either on FET- or DOTATOC-PET were contoured and compared with each other as well with $\mathrm{GTV}_{\mathrm{CT} / \mathrm{MR} \text {. }}$.

Results: Every tumour showed typical enhancement of DOTATOC, but two meningiomas remained FET-negative. The mean relative overlap volume of GTV FET and GTV DOTATOC was only $41.9 \%$ and there was a significantly stronger correlation between $\mathrm{GTV}_{\mathrm{CT} / \mathrm{MRI}}$ and $\mathrm{GTV}_{\text {DOTATOC }}$ than between GTV $\mathrm{VTTMRI}_{\text {and }}$ GTV FET.

Conclusions: Further investigations are necessary to clarify the minor conformity of DOTATOC- and FET-PET in meningiomas. Because of the receptor targeting, DOTATOC is known to be more specific for meningiomas and will remain the standard in our institution with the known limitation in areas nearby the pituitary gland.
\end{abstract}

Keywords: Meningioma, PET, DOTATOC, FET, Radiation therapy

\section{Background}

Over the last decades radiation therapy (RT) has been established as a successful, safe and effective treatment of intracranial meningiomas. Because of their mostly benign character, a highly conformal RT is essential to avoid radiation-induced side effects. Better and better high precision RT techniques have been developed in the past like Fractionated Stereotactic Radiotherapy, Intensity Modulated Radiotherapy, Radiosurgery as well as Particle Therapy with protons or carbon ions [1-5], resulting in high control rates with very low rates of side effects $[1,6-10]$.

However the more precise radiation techniques become, the more an accurate delineation of the gross

\footnotetext{
* Correspondence: Stephanie.Combs@tum.de

${ }^{1}$ Department of Radiation Oncology, Klinikum rechts der Isar, Technische Universität München (TUM), Ismaninger Str. 22, 81675 Munich, Germany Full list of author information is available at the end of the article
}

tumour volume (GTV) is essential. Distinguishing between tumour and surrounding normal tissue can be difficult even by combining computed tomography (CT) and magnetic resonance imaging (MRI) e.g. for evaluation of bony involvement or the dural tail [11, 12]. Furthermore, strong enhancement of contrast fluid in CT- and MRI-scans is seen both in meningioma cells and neighbouring normal tissue like the cavernous or sagittal sinus. Therefore a trimodal image fusion using CT, MRI and positron emission tomography (PET) has been established for treatment planning of meningiomas [13-18]. PET-tracers based either on amino acids as $\left[{ }^{11} \mathrm{C}\right]$-Methionine (MET) and $\left[2-{ }^{18} \mathrm{~F}\right]$-fluoro-L-tyrosine (TYR) or the somatostatin receptor 2 (SSTR2) ligand $\left[{ }^{68} \mathrm{Ga}\right]$-DOTA-D Phe 1-3Tyr3-Octreotide (DOTATOC) were shown to be beneficial for RT-planning, since 
these show very high meningioma to background ratios [13-21].

Nevertheless, there are also limitations for MET and DOTATOC: An onsite cyclotron is required to produce MET because of the short physical half-life of $20 \mathrm{~min}$ for ${ }^{11} \mathrm{C}$. Due to this fact its use is restricted to a small number of research centers $[15,22]$. DOTATOC is easier to handle than MET, but since the pituitary gland also expresses SSTR2 a high uptake of DOTATOC in the sella turcica is physiological and therefore an intrasellar invasion by meningioma cells cannot be differentiated [21]. In such cases, close correlation with CT and MRI is necessary to distinguish meningioma from pituitary tissue.

$\mathrm{O}-\left(2-\left[{ }^{18} \mathrm{~F}\right]\right.$ fluoroethyl)-L-tyrosine (FET) is another amino acid-based PET-tracer. In contrast to MET, PETscans with FET can be performed even by departments distant from a cyclotron, since half-life of ${ }^{18} \mathrm{~F}$ is more than 5 times longer. Although the uptake mechanisms of MET and FET differ, a close correlation of the intensity of tracer uptake was described in tumoral as well as in non-tumoral cerebral lesions [22, 23]. Moreover, advantageously in comparison to DOTATOC there was no increased uptake in cells of the pituitary gland reported. Therefore, FET was hypothesized to be a superior tracer in the imaging of meningiomas, although the clinical experience of FET-PET in meningioma patients is still limited [24, 25].

Only recently, an EANO taskforce generated a guideline for diagnosis and treatment of meningiomas [26]. However, inter-institutional differences in the use of PET-tracers for the treatment planning process for meningiomas exist. So the aim of this study was to evaluate the influence of these different types of PETtracers on GTV-definition in meningiomas.

\section{Methods \\ Patients}

Between October 2010 and February 201221 patients suffering from intracranial meningiomas underwent neuroimaging including CT, MRI, FET- and DOTATOCPET for treatment planning at the Department of Radiation Oncology in Heidelberg, Germany.

All four examinations were performed in a mean period of 7.7 days (range from 2 to 27 days). The mean time lag of the performances of DOTATOC- and FETPET-scans was 4.5 days (range from 1 to 21 days).

Nineteen of the 21 patients (90.5\%) had undergone surgery for their meningioma in the past and therefore a histological grading could be obtained. Six patients (28.6\%) had had even more than one surgical resection in their lifetime. The median interval between the last surgical resection and the performance of the CT-, MRI- and PET-scans evaluated in this study was
45.4 months (range from 3 to 204 months). For patient characteristics see Table 1 .

The performance of this study was approved by the local ethics committee (Ethikkommission Medizinische Fakultät Heidelberg).

\section{Imaging}

All patients received an individually formed fixation device including scotch-cast masks for seven patients (33.3\%) or thermoplastic masks for 14 patients (66.7\%) to immobilize the patient's head as described before $[2,27]$.

CT-scans were performed using a Siemens Sensation 4 (Siemens, Erlangen, Germany) with slice-thickness of $3 \mathrm{~mm}$ after application of contrast medium $(1,5 \mathrm{ml} / \mathrm{kg}$ body weight, Ultravist 300, Bayer, Leverkusen, Germany).

MRI was performed in most patients using a $3.0-\mathrm{T}$ MR scanner (Siemens Trio or Siemens Verio). T1-W images were obtained after administration of contrast fluid according to the body weight (Magnograf, Marotrast $\mathrm{GmbH}$, Jena, Germany or Gadovist, Bayer, Leverkusen, Germany) with slice-thickness of $1.3 \mathrm{~mm}$ (TR 1.710; TE 4.04). Three patients received T1-W images using a 1.5-T MR scanner (Siemens Symphonie) with slice-thickness of $3 \mathrm{~mm}$ (TR 13; TE 4.7) after application of contrast medium (MultiHance, Bracco IMAGING, Konstanz, Germany) since a 3.0-T MR scanner was not eligible or not available in a timely manner.

Table 1 Patient characteristics

\begin{tabular}{|c|c|c|}
\hline Age & [Years] & \\
\hline Median age & 50 & \\
\hline Range & $26-75$ & \\
\hline Sex & {$[n]$} & [\%] \\
\hline Female & 16 & 76 \\
\hline Male & 5 & 24 \\
\hline \multicolumn{3}{|l|}{ Main anatomical localisation } \\
\hline Skull base & 17 & 81 \\
\hline Parasagittal region & 3 & 14 \\
\hline Convexity & 1 & 5 \\
\hline \multicolumn{3}{|l|}{ Treatment concept } \\
\hline RT after incomplete resection & 3 & 14 \\
\hline RT at tumour progression & 16 & 76 \\
\hline Primary RT & 2 & 10 \\
\hline Surgery in past & 19 & 91 \\
\hline \multicolumn{3}{|l|}{ Histological subtype } \\
\hline WHO grade I & 12 & 57 \\
\hline WHO grade $\|$ & 6 & 29 \\
\hline WHO grade III & 1 & 5 \\
\hline Unknown & 2 & 10 \\
\hline
\end{tabular}

$R T$ radiation therapy 
${ }^{68} \mathrm{Ga}$-DOTATOC was produced as previous published and injected intravenously as bolus [28]. The mean administered activity was $168 \mathrm{MBq}$ (range: 102-197 MBq) and the peptide quantity was $12.5 \mu \mathrm{g}$ DOTATOC in all preparations. The examination was done with a Biograph$6 \mathrm{PET} / \mathrm{CT}$ (Siemens). Approximately $30 \mathrm{~min}$ after injection a diagnostic CT of the head was performed (250 $\mathrm{mAs}, 110 \mathrm{kV}$, slice collimation $6 \times 2 \mathrm{~mm}$, slice-thickness $3 \mathrm{~mm}$ Full Width at Half Maximum (FWHM), 0.9 Pitch) which was also used for attenuation correction. One bed position with a $15.5 \mathrm{~cm}$ field of view was acquired with 10 min scan time and reconstructed with an ordered subset expectation maximization (OSEM) algorithm with four iterations of 16 subsets and Gauss filtering to achieve images with a matrix size of $256 \times 256$ and an in-plane spatial resolution of $5 \mathrm{~mm}$ FWHM.

${ }^{18} \mathrm{~F}$-FET was obtained commercially from IASON (Graz, Austria) and was injected intravenously as bolus with a mean activity of $184 \mathrm{MBq}$ (range: $158-218 \mathrm{MBq}$ ). The examination was also done with the Biograph-6 PET/CT. A native low-dose CT $(60 \mathrm{mAS}, 130 \mathrm{kV}$, slice collimation 6x3mm, slice-thickness $5 \mathrm{~mm}$ FWHM, 1.5 Pitch) was done for attenuation correction. The emission was acquired as a dynamic scan and then a static frame covering 20-40 min after injection was reconstructed with the OSEM algorithm (two iterations of 16 subsets, Gauss filtering, matrix size of $256 \times 256$, in-plane spatial resolution $5 \mathrm{~mm}$ FWHM). The uptake of FET and DOTATOC was quantified by standardized uptake values (SUVs).

\section{Image fusion, GTV delineation and quantitative analysis of tumour volumes}

Using Siemens COHERENCE Dosimetrist (Siemens Medical solutions, Concord, CA) image fusion was performed by matching contrast enhanced CT-images with contrast enhanced MRI-T1-W images, FET- and DOTATOC-PET images. Because of a high accuracy of the used image fusion method, errors in coregistration were reduced to a minimum. Therefore, a head fixation device for MRI- and PET-scans was not stringently necessary.

At first tumour extension was delineated by cooperation of two experienced radiooncologists after imagefusion of CT and MRI in each patient, resulting in $\mathrm{GTV}_{\mathrm{CT} / \mathrm{MRI}}$, that included any macroscopic tumour suspicious tissue in contrast enhanced CT and MRI. In a second step in every patient distinct GTVs based on FET- and DOTATOC-PET were contoured $\left(\mathrm{GTV}_{\mathrm{FET}}\right.$, $\left.\mathrm{GTV}_{\text {DOTATOC}}\right)$ by the same advanced radiooncologists in cooperation with an experienced nuclear radiologist respectively. Since a general cut-off value for the SUVs of DOTATOC as well as FET is missing so far, the PETwindow levels were adjusted so that the PET-delineation was in accordance with the $\mathrm{GTV}_{\mathrm{CT} / \mathrm{MRI}}$ on slices with a clear differentiation of tumour borders on CT and MRI as described by Thorwarth et al. [18] before.

Afterwards GTV $_{\text {FET }}$ was compared with GTV $_{\text {DOTATOC }}$ as well as $G_{T V} V_{\mathrm{FET}}$ and $\mathrm{GTV}_{\mathrm{DOTATOC}}$ were compared with $\mathrm{GTV}_{\mathrm{CT} / \mathrm{MRI}}$. The relative overlap and non-overlap volumes of these GTVs were calculated as described before by Astner et al. [13] (Fig. 1): In detail, the data analysis of each volume using the results of the fusion images was performed. Then, a common volume included the volumes derived from MRI and CT showing PET-uptake. Thereafter, volumes of PET uptake outside the volume $\mathrm{GTV}_{\mathrm{CT}}$ / MRI was determined and rated as an increase in volume of $\mathrm{GTV}_{\mathrm{CT} / \mathrm{MRI}}$. Lastly, the volume of GTV $\mathrm{CT}_{\mathrm{CT} / \mathrm{MRI}}$ outside the PET-changes was defined as an increase in $G \mathrm{VV}_{\mathrm{PET}}$.

All GTVs shown in this study were retrospectively delineated for research only to avoid systemic interpersonal differences since the original 21 GTVs used for treatment planning were contoured by different radiooncologists. The original irradiation plan for patient treatment was calculated with DOTATOC-based GTVs mainly since DOTATOC is the standard tracer in our centre for meningioma radiotherapy planning and there was no large experience with FET-PET in meningiomas so far.

\section{Statistics}

Statistical analyses were performed with IBM SPSS Statistics Version 20 (IBM, Armonk, NY). The relative corresponding volumes were statistically tested using the

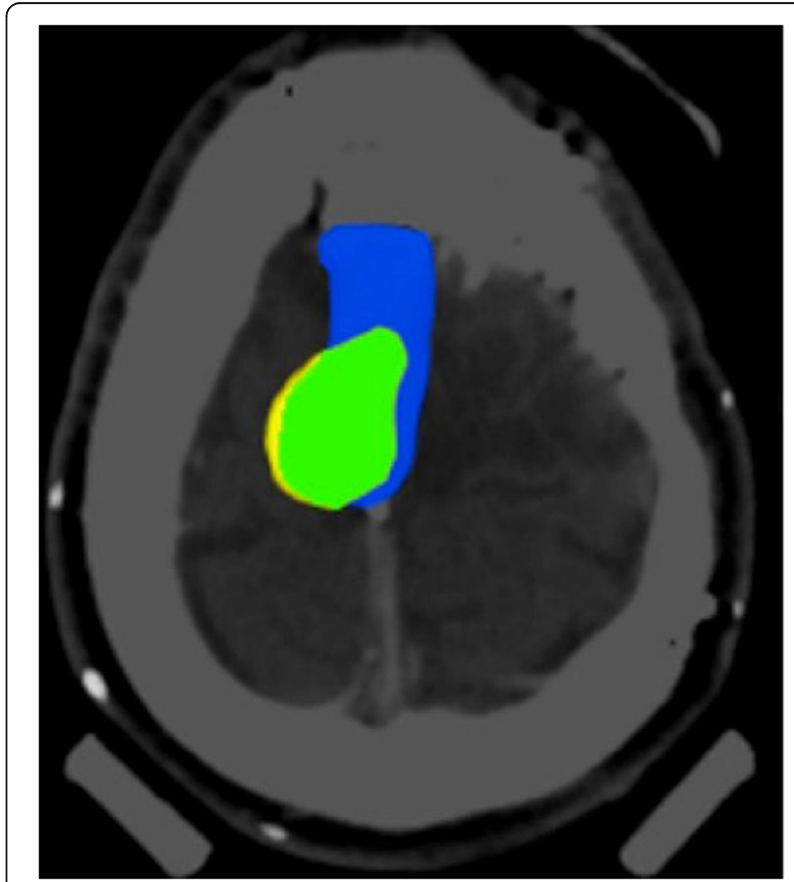

Fig. 1 Corresponding volume (green) of GTV GTV FET (yellow) of one patient. For more details of this patient including CT-, MRI-, DOTATOC- and FET-PET-images see Fig. 5a 
T-test depending on the type of tracer. The relative corresponding volumes of $\mathrm{GTV}_{\text {DOTATOC }}$ and GTV $\mathrm{FET}_{\mathrm{FET}}$ were tested depending on WHO-grade. All statistical tests were performed at a $5 \%$ level of significance.

\section{Results}

In all patients meningiomas could be clearly identified on CT, MRI and DOTATOC-PET. In contrast, the sole anaplastic meningioma showed no enhancement of FET and another patient with two meningiomas of the skull base had one FET-positive and one FET-negative lesion.

The mean volumes of $\mathrm{GTV}_{\mathrm{CT} / \mathrm{MRI}}, \mathrm{GTV}_{\mathrm{FET}}$ and $\mathrm{GTV}_{\text {DОТАTOC }}$ were $53.9 \mathrm{ccm}$ (range $0.46-179.5 \mathrm{ccm}$ ), $34.7 \mathrm{ccm}$ (range $0-117.6 \mathrm{ccm})$ and $39.4 \mathrm{ccm}(0.2-$ $134.2 \mathrm{ccm}$ ) respectively (Fig. 2). The $\mathrm{GTV}_{\text {DОтАтOC }}$ enclosed a mean minimum relative SUV of $56.1 \%$ (standard deviation 0.02) and the $\mathrm{GTV}_{\mathrm{FET}}$ a mean minimum relative SUV of $65.9 \%$ (standard deviation $0.05)$ respectively. The mean minimal SUV-lesion to normal ratio (LNR) for the delineated $\mathrm{GTV}_{\mathrm{FET}}$ was 1.5 (standard deviation 0.45).

The mean relative corresponding volume of $\mathrm{GTV}_{\mathrm{FET}}$ and $\mathrm{GTV}_{\text {DOTATOC }}$ was $41.9 \%$ only (range $0-61.6 \%$ ). In WHO Grade I meningiomas there was a mean relative corresponding volume of $45.9 \%$ whereas in atypical meningiomas it was less with $39.9 \%(p>0.05)$. Since there was no enhancement of FET in the sole anaplastic meningioma, a relative corresponding volume of both PET-CTs could be calculated neither for this patient nor for anaplastic meningiomas (Fig. 3).

There was a significantly stronger correlation between $\mathrm{GTV}_{\mathrm{CT} / \mathrm{MRI}}$ and $\mathrm{GTV}_{\text {DOTATOC }}$ than between $\mathrm{GTV}_{\mathrm{CT} / \mathrm{MRI}}$

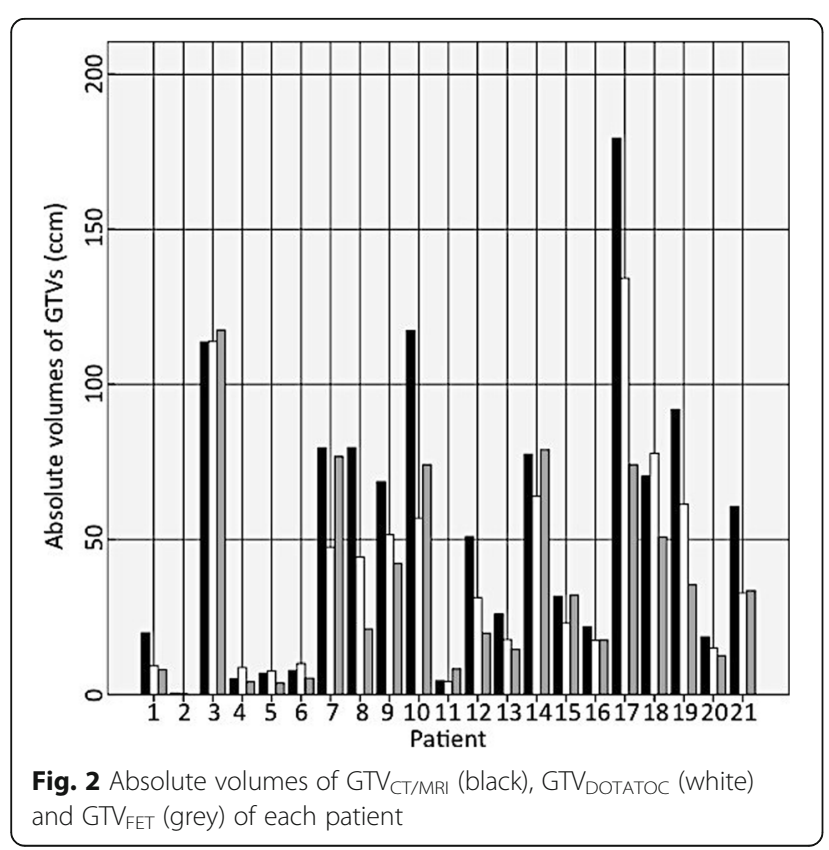

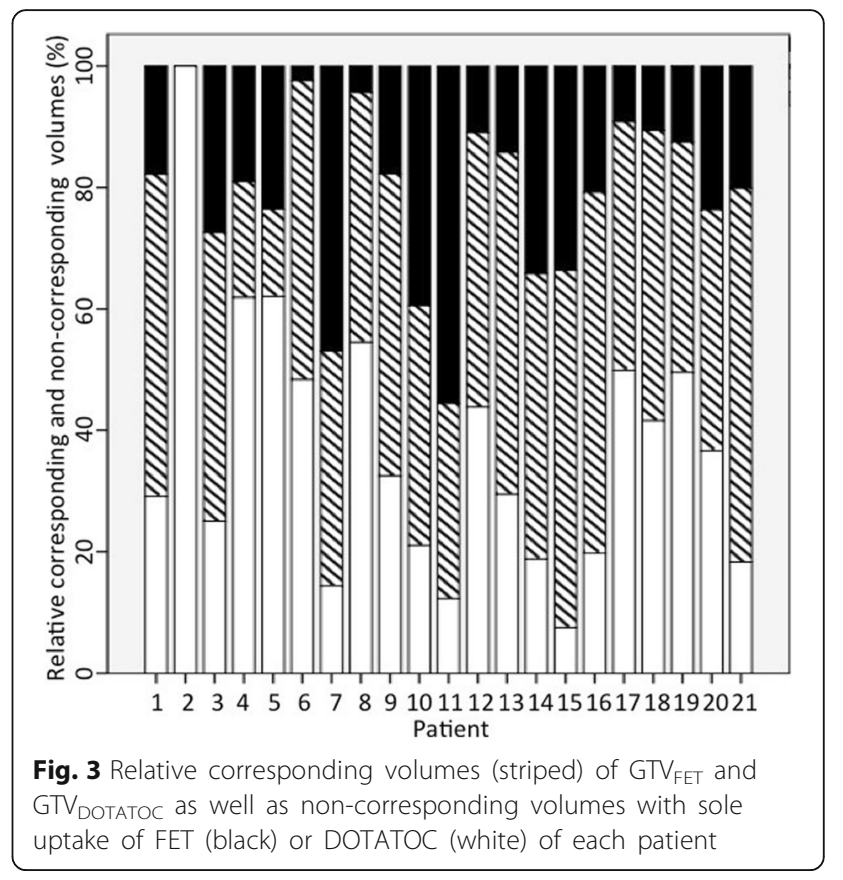

and $\mathrm{GTV}_{\mathrm{FET}}$ with mean relative corresponding volumes of $52.0 \%$ and $36.8 \%$, respectively $(p<0.001)$.

\section{Discussion}

This is the first report of an intraindividual comparison of an amino acid-based PET and DOTATOC- PET in patients with intracranial meningiomas. Furthermore, we present the largest population of patients suffering from intracranial meningiomas that were examined by FET-PET so far.

A trimodal image fusion of CT, MRI and PET is well established in the treatment planning process for RT of intracranial meningiomas. The MRI provides a high accuracy especially in soft tissues. The CT is necessary for accurate computation of radiation doses and helps to estimate the involvement of bony structures. Additional information about tumour extension and biology are presented by PET-scans [15]. The widely used PET tracer ${ }^{18}$ F-fluorodeoxy-glucose (FDG) mostly fails for brain tumour imaging because of the physiologically high metabolism of glucose in the cerebral cortex. In contrast to FDG, the amino acid-based tracers and DOTATOC have been shown to offer high meningioma to background ratios.

Milker-Zabel et al. [16] reported a significant modification of PTV for FSRT in 19 of 26 patients (73\%) combining MRT and CT with DOTATOC-PET for treatment planning of meningiomas. Similar results were shown by Gehler et al. [14]. The DOTATOC-PET gave additional information for the GTV-delineation in IMRT-planning in $65 \%$ of their cases. By combining MET-PET with CT and MRI Astner et al. [13] demonstrated an influence 
on GTV in $90.6 \%$. In most cases of their study this resulted in a smaller GTV. By addition of MET-PET they could better discriminate non-tumorous structures as the cavernous sinus, the sella region or meningeal reaction after previous surgery. Furthermore a reduction of the interobserver variability in target volume definition of meningiomas was reported by Grosu et al. [15] using MET-PET for treatment planning. Rutten et al. [17] found discrepancies between MRI and TYR-PET in $46 \%$ of intracranial meningiomas in their study. From these cases $83 \%$ of the PET lesions extended beyond the MRI lesion. In summary a benefit for additional information regarding the extension of meningiomas was shown for DOTATOC as well as for amino acid-based tracers $[16,17,21,29]$. Up to now there was a missing evidence supporting the superiority of one of these tracers [14].

FET-PET was shown to be more accurate than FDGPET for detecting malignant brain lesions [30]. To date experiences for FET-PET in meningiomas are rare [24, 25]. In a PET-comparison study of brain lesions Lau et al. [30] included only one anaplastic meningioma. In other studies comparing FET- and MET-PET of intracranial malignancies, no patient suffering from a meningioma was investigated $[22,23]$. Although a close correlation of the intensity of tracer uptake was described for the amino acidbased tracers MET and FET, the uptake mechanism and further intracellular pathways of these two are quite different $[22,23]$ : MET is mainly transported by the Ltransport-system, a bi-directional amino acid carrier, as well as by the A-system and enters several biochemical pathways [17]. It is utilized for protein synthesis, converted to S-adenosyl-methionine e.g. as precursor for polyamine synthesis or metabolised by decarboxylation [23]. As an analogue of tyrosine the uptake of FET is thought to be selectively mediated by LAT2, a Ltransporter subtype. Moreover it is not incorporated into proteins or otherwise metabolized [23]. However, although the transport mechanisms of FET seem to be rather specific, the previously reported clinical results concerning neuroimaging seem rather comparable to MET [22, 23].

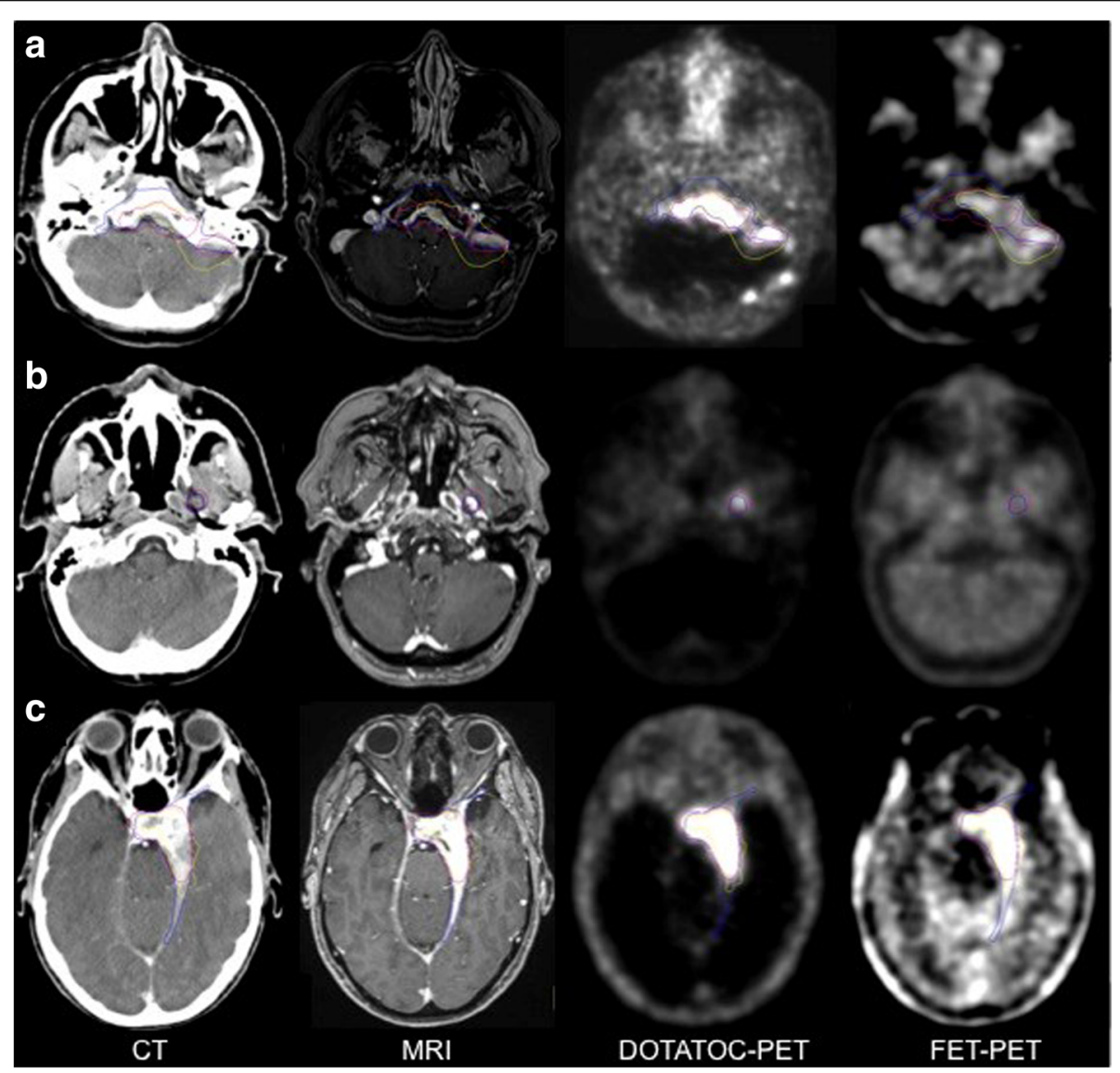

Fig. 4 Delineated GTVs of three more patients based on CT and MRI (blue), DOTATOC-PET (purple) or FET-PET (yellow): Meningioma and parts of the neighbouring brain tissue show similar uptake in FET-PET $(A+C)$. A group of three small meningiomas next to the dorsal left cerebellar hemisphere is well visible in MRI and DOTATOC-PET but shows no uptake of FET (a). An extracranial branch of the meningioma is not visible in FET-PET since it is neighbouring muscular structures (b). The FET-PET clearly shows the complete tumour infiltration of the pituitary gland but also a strong uptake at the sagittal venous sinus and temporal muscles (c) 
Our data shows a significantly better correlation between CT/MRI and DOTATOC-PET than between CT/MRI and FET-PET. Because of the receptor targeting, DOTATOC is known to be more specific for meningiomas with the known limitation in areas close to the parasellar region since the pituitary gland expresses SSTR2 [21]. In our study, nine of 21 meningiomas were neighbouring this region, so in these patients DOTATOC failed to discriminate between meningioma and pituitary gland. When a clear delineation between meningioma and pituitary gland was not possible we included this organ in the $\mathrm{GTV}_{\text {DOTATOC }}$ for the evaluation of this study. Compared to DOTATOC, FET is known not to accumulate in the pituitary gland. Despite this advantage, we also have to report about limitations of FET-PET. Due to a low urinary excretion of FET (22\% after $5 \mathrm{~h}$ ), the concentration in blood compartment was found to be relatively high especially in the first hour after injection [31]. Therefore, a visualisation of large vessels as venous sinus can be misinterpreted and the clear delineation of a neighbouring meningioma becomes difficult (Fig. 4c). Moreover, in cases where meningioma cells are neighbouring muscular structures there is more "noise" of the surrounding tissue in FET-PET which results in a reduced discrimination of the meningioma's branches growing through the holes of the skull base to extra cranial sites [31] (Fig. 4b). In several patients the $G_{T V}$ exceeded the $G_{T V}$ DOTATOC as well as the $\mathrm{GTV}_{\mathrm{CT} / \mathrm{MRT}}$ by including the neighbouring cerebral cortex (Fig. 4a and c, 5a). It was hypothesized before that an enlarged PET-GTV could be caused by a microscopical infiltration of meningioma cells into surrounding tissues following vascular structures or cranial nerves [17]. Limitations of manual tumour contour delineation or suboptimal windowing of PET-images could be other explanations for slight discrepancies of the different GTV [22]. However compared to DOTATOC we observed a much stronger uptake of FET in the normal cerebral cortex located close by as well as distant from the meningioma. This was also reflected in a mean minimum LNR of FET of 1.5 with a standard deviation of 0.45 .

Moreover we have to report about two completely and several partially FET-negative meningiomas showing no FET-uptake in areas highly tumour-suspicious in CT, MRI and DOTATOC-PET (Fig. 5). An explanation could

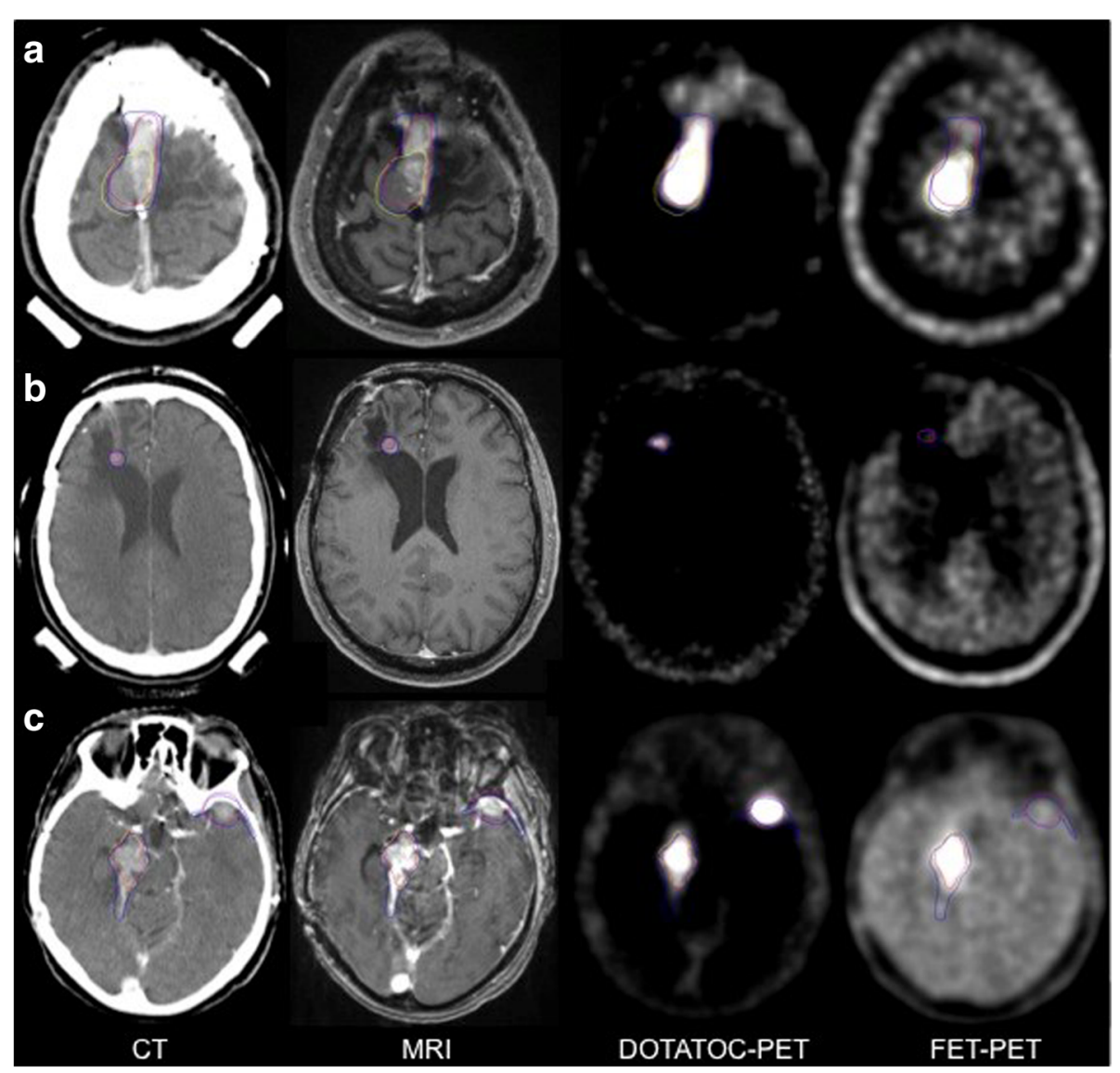

Fig. 5 Delineated GTVs of three patients based on CT and MRI (blue), DOTATOC-PET (purple) or FET-PET (yellow): Meningioma is partially FET-negative (a), Meningioma is completely FET-negative (b). Patient $\mathrm{C}$ (c) is suffering from two meningiomas, one FET-positive and the other FET-negative. All meningiomas are clearly visible in DOTATOC-PET, CT and MRI 
be that amino acid-based tracers are known to provide functional aspects of the activity of meningioma cells and for FET-negative areas maybe a lesser activity up to no activity of growth is to hypothesize. For gliomas it was found that accumulation of MET correlates better with histological tumour spread than CT or MRI [31]. For MET-uptake in meningiomas a significant correlation to Ki-67 index was shown [23] and Gudjonsson et al. [32] could demonstrate that a proton radiation of meningiomas led to a reduction of MET uptake of 19.4\%. Potentially in further studies GTV-delineation could be restricted to FET-PET positive parts of meningioma only. But since both FET-negative meningiomas of our study were grown in size in further follow up, this modus operandi seems too hazardous, potentially underestimating the actual extension of the tumour. So apart from the parasellar region DOTATOC seems to be more sensitive and also more specific for the delineation of meningioma tissue than FET.

In comparison to other studies that investigated the effect of amino acid-based tracers in treatment planning of meningiomas we included much more histologically proven high-grade meningiomas: one meningioma grade III and six meningiomas grade II, in total 33.4\% (Rutten et al.: $0 \%(0 / 11)$ [17], Grosu et al. 0\% (0/10) [15] and Astner et al. $3.1 \%$ only (1/32) [13]). This is remarkable since both patients with FET-negative meningiomas in our study were suffering from a high-grade meningioma and the missing FET-uptake could possibly be caused by a different biology in comparison to low-grade meningiomas.

Thus, the data from the present analysis describes differential tracer uptake of DOTATOC and FET in meningiomas as a first intraindividual comparison. To date, the questions whether one or the other tracer is superior cannot definitely be answered. Further investigations are necessary to clarify the minor congruence of DOTATOC- and FET-PET in meningiomas. Histological und molecular biological examinations of bioptic material taken from areas with different tracer uptake could help to understand the reasons. Alternatively, clinical studies could evaluate the outcome after irradiation of the FET-positive tumour parts only. However, as highly tumour suspicious areas remained FET-negative and were grown in size in further follow-up, a restriction to FET-positive areas cannot be recommended in treatment planning of meningiomas so far. Although a close correlation between the uptake of the amino acid-based tracers MET and FET was described in literature, our results of the FET-uptake in meningiomas should not be generalized for other amino acid-based PET-tracers. Despite the known limitations in the area close to the pituitary gland, DOTATOC-PET remains to be our in-house standard, as DOTATOC is known to be more specific for meningiomas and as we have gained a lot of experience with DOTATOC-based treatment planning for irradiation of meningiomas over the last decades [16, 29, 33, 34].

\section{Conclusion}

Volumes based on FET and DOTATOC in meningiomas can be hetereogeneous based on the tracer applied. In general DOTAOTC-PET shows the best overlap with $\mathrm{MR} / \mathrm{CT}$. Thus, based on the data from this analysis in concordance with published data DOTATOC-PET is recommended for treatment planning of meningiomas and should be implemented when available.

\section{Abbreviations \\ CT: Computed tomography; FDG: ${ }^{18}$ F-fluorodeoxy-glucose; FET: O-(2- $\left[{ }^{18}\right.$ F]fluoroethyl)-L-tyrosine; FWHM: Full Width at Half Maximum; GTV: Gross tumour volume; MET: Methionine; MRI: Magnetic resonance imaging; OSEM: Ordered subset expectation maximization; PET: Positron emission tomography; RT: Radiation therapy; SSTR2: Somatostatin receptor 2; SUV: Standardized uptake values; TYR: Tyrosine; DOTATOC: [ ${ }^{68}$ Ga]-DOTA-D Phe 1-3Tyr3-Octreotide}

\section{Acknowledgements \\ Not applicable.}

\section{Funding}

This work was performed in the framework of the German Research Foundation, Klinische Forschergruppe Schwerionentherapie (DFG, KFO 214).

Availability of data and materials

The data supporting the conclusions of this article are included within the article.

\section{Authors' contributions \\ SEC, JD, AD, JOD, CK, UH, DH, SR and FG performed the examinations and treated the patients. SEC and JOD set up the design of the analyses. SEC obtained ethic's vote. JOD and SEC evaluated the data and drafted the manuscript. JD, AD, CK, UH, DH, SR and FG reviewed and discussed the data, and optimized the manuscript. All co-authors read and approved the paper.}

Ethics approval and consent to participate

The performance of this study was approved by the local ethics committee (Ethikkommission Medizinische Fakultät Heidelberg).

Consent for publication

Not applicable.

\section{Competing interests}

The authors declare that they have no competing interests.

\section{Publisher's Note}

Springer Nature remains neutral with regard to jurisdictional claims in published maps and institutional affiliations.

\section{Author details}

${ }^{1}$ Department of Radiation Oncology, Klinikum rechts der Isar, Technische Universität München (TUM), Ismaninger Str. 22, 81675 Munich, Germany. ${ }^{2}$ Department of Radiation Oncology, University Hospital of Heidelberg, Im Neuenheimer Feld 400, 69120 Heidelberg, Germany. ${ }^{3}$ Department of Nuclear Medicine, University Hospital of Heidelberg, Im Neuenheimer Feld 400, 69120 Heidelberg, Germany. ${ }^{4}$ Institute for Innovative Radiotherapy (iRT), Department of Radiation Sciences (DRS), Helmholtz Zentrum München, 85764 Oberschleißheim, Germany. ${ }^{5}$ Deutsches Konsortium für Translationale Krebsforschung (DKTK), Partner Site Munich, Munich, Germany. 
Received: 25 August 2017 Accepted: 27 October 2017

Published online: 06 November 2017

\section{References}

1. Combs SE, Hartmann C, Nikoghosyan A, Jakel O, Karger CP, Haberer T, von Deimling A, Munter MW, Huber PE, Debus J, Schulz-Ertner D. Carbon ion radiation therapy for high-risk meningiomas. Radiother Oncol. 2010;95:54-9.

2. Combs SE, Sterzing F, Uhl M, Habl G, Schubert K, Debus J, Herfarth K. Helical tomotherapy for meningiomas of the skull base and in paraspinal regions with complex anatomy and/or multiple lesions. Tumori. 2011;97:484-91.

3. Milker-Zabel S, Zabel-du Bois A, Huber P, Schlegel W, Debus J. Fractionated stereotactic radiation therapy in the management of benign cavernous sinus meningiomas : long-term experience and review of the literature. Strahlenther Onkol. 2006;182:635-40.

4. Nakamura JL, Pirzkall A, Carol MP, Xia P, Smith V, Wara WM, Petti PL, Verhey $\sqcup$, Sneed PK. Comparison of intensity-modulated radiosurgery with gamma knife radiosurgery for challenging skull base lesions. Int J Radiat Oncol Biol Phys. 2003:55:99-109.

5. Pirzkall A, Debus J, Haering P, Rhein B, Grosser KH, Hoss A, Wannenmacher M. Intensity modulated radiotherapy (IMRT) for recurrent, residual, or untreated skull-base meningiomas: preliminary clinical experience. Int J Radiat Oncol Biol Phys. 2003;55:362-72.

6. Alheit H, Saran FH, Warrington AP, Rosenberg I, Perks J, Jalali R, Shepherd S, Beardmore C, Baumert B, Brada M. Stereotactically guided conformal radiotherapy for meningiomas. Radiother Oncol. 1999;50:145-50.

7. Minniti G, Amichetti M, Enrici RM. Radiotherapy and radiosurgery for benign skull base meningiomas. Radiat Oncol. 2009;4:42

8. Milker-Zabel S, Zabel A, Schulz-Ertner D, Schlegel W, Wannenmacher M, Debus J. Fractionated stereotactic radiotherapy in patients with benign or atypical intracranial meningioma: long-term experience and prognostic factors. Int J Radiat Oncol Biol Phys. 2005;61:809-16.

9. Selch MT, Ahn E, Laskari A, Lee SP, Agazaryan N, Solberg TD, Cabatan-Awang C, Frighetto L, Desalles AA. Stereotactic radiotherapy for treatment of cavernous sinus meningiomas. Int J Radiat Oncol Biol Phys. 2004;59:101-11.

10. Combs SE, Adeberg S, Dittmar JO, Welzel T, Rieken S, Habermehl D, Huber $P E$, Debus J. Skull base meningiomas: long-term results and patient selfreported outcome in 507 patients treated with fractionated stereotactic radiotherapy (FSRT) or intensity modulated radiotherapy (IMRT). Radiother Oncol. 2013;106:186-91.

11. Guermazi A, Lafitte F, Miaux Y, Adem C, Bonneville JF, Chiras J. The dural tail sign-beyond meningioma. Clin Radiol. 2005;60:171-88.

12. Pieper DR, Al-Mefty O, Hanada Y, Buechner D. Hyperostosis associated with meningioma of the cranial base: secondary changes or tumor invasion. Neurosurgery. 1999:44:742-6. discussion 746-747

13. Astner ST, Dobrei-Ciuchendea M, Essler M, Bundschuh RA, Sai H, Schwaiger M, Molls M, Weber WA, Grosu AL. Effect of 11C-methionine-positron emission tomography on gross tumor volume delineation in stereotactic radiotherapy of skull base meningiomas. Int J Radiat Oncol Biol Phys. 2008;72:1161-7.

14. Gehler B, Paulsen F, Oksuz MO, Hauser TK, Eschmann SM, Bares R, Pfannenberg C, Bamberg M, Bartenstein P, Belka C, Ganswindt U. [68Ga]-DOTATOC-PET/CT for meningioma IMRT treatment planning. Radiat Oncol. 2009:4:56.

15. Grosu AL, Weber WA, Astner ST, Adam M, Krause BJ, Schwaiger M, Molls M, Nieder C. 11C-methionine PET improves the target volume delineation of meningiomas treated with stereotactic fractionated radiotherapy. Int $J$ Radiat Oncol Biol Phys. 2006;66:339-44.

16. Milker-Zabel S, Zabel-du Bois A, Henze M, Huber P, Schulz-Ertner D, Hoess A, Haberkorn U, Debus J. Improved target volume definition for fractionated stereotactic radiotherapy in patients with intracranial meningiomas by correlation of CT, MRI, and [68Ga]-DOTATOC-PET. Int J Radiat Oncol Biol Phys. 2006;65:222-7.

17. Rutten I, Cabay JE, Withofs N, Lemaire C, Aerts J, Baart V, Hustinx R. PET/CT of skull base meningiomas using 2-18F-fluoro-L-tyrosine: initial report. J NuCl Med. 2007:48:720-5.

18. Thorwarth D, Henke G, Muller AC, Reimold M, Beyer T, Boss A, Kolb A, Pichler B, Pfannenberg C. Simultaneous 68Ga-DOTATOC-PET/MRI for IMRT treatment planning for meningioma: first experience. Int J Radiat Oncol Biol Phys. 2011;81:277-83.

19. Thorwarth D, Muller AC, Pfannenberg C, Beyer T. Combined PET/MR imaging using (68)Ga-DOTATOC for radiotherapy treatment planning in meningioma patients. Recent Results Cancer Res. 2013;194:425-39.
20. Graf R, Nyuyki F, Steffen IG, Michel R, Fahdt D, Wust P, Brenner W, Budach V, Wurm R, Plotkin M. Contribution of 68Ga-DOTATOC PET/CT to target volume delineation of skull base meningiomas treated with stereotactic radiation therapy. Int J Radiat Oncol Biol Phys. 2013;85:68-73.

21. Henze M, Schuhmacher J, Hipp P, Kowalski J, Becker DW, Doll J, Macke HR, Hofmann M, Debus J, Haberkorn U. PET imaging of somatostatin receptors using [68GA]DOTA-D-Phe1-Tyr3-octreotide: first results in patients with meningiomas. J Nucl Med. 2001;42:1053-6.

22. Grosu AL, Astner ST, Riedel E, Nieder C, Wiedenmann N, Heinemann F, Schwaiger M, Molls M, Wester HJ, Weber WA. An interindividual comparison of O-(2-[18F]fluoroethyl)-L-tyrosine (FET)- and L-[methyl-11C]methionine (MET)-PET in patients with brain gliomas and metastases. Int J Radiat Oncol Biol Phys. 2011;81:1049-58.

23. Weber WA, Wester HJ, Grosu AL, Herz M, Dzewas B, Feldmann HJ, Molls M, Stocklin G, Schwaiger M. O-(2-[18F]fluoroethyl)-L-tyrosine and L-[methyl$11 \mathrm{C}]$ methionine uptake in brain tumours: initial results of a comparative study. Eur J Nucl Med. 2000;27:542-9.

24. Cornelius JF, Langen KJ, Stoffels G, Hanggi D, Sabel M, Jakob Steiger H. Positron emission tomography imaging of meningioma in clinical practice: review of literature and future directions. Neurosurgery. 2012;70:1033-41. discussion 1042

25. Cornelius JF, Slotty PJ, Stoffels G, Galldiks N, Langen KJ, Steiger HJ. 5Aminolevulinic acid and (18)F-FET-PET as metabolic imaging tools for surgery of a recurrent Skull Base Meningioma. J Neurol Surg B Skull Base. 2013;74:211-6.

26. Goldbrunner R, Minniti G, Preusser M, Jenkinson MD, Sallabanda K, Houdart E, von Deimling A, Stavrinou P, Lefranc F, Lund-Johansen M, et al. EANO guidelines for the diagnosis and treatment of meningiomas. Lancet Oncol. 2016;17:e383-91.

27. Debus J, Engenhart-Cabillic R, Knopp MV, Schad LR, Schlegel W, Wannenmacher M. Image-oriented planning of minimally invasive conformal irradiation of the head-neck area. Radiologe. 1996;36:732-6.

28. Otte A, Jermann E, Behe M, Goetze M, Bucher HC, Roser HW, Heppeler A, Mueller-Brand J, Maecke HR. DOTATOC: a powerful new tool for receptor-mediated radionuclide therapy. Eur J Nucl Med. 1997;24:792-5.

29. Henze M, Dimitrakopoulou-Strauss A, Milker-Zabel S, Schuhmacher J, Strauss LG, Doll J, Macke HR, Eisenhut M, Debus J, Haberkorn U. Characterization of 68Ga-DOTA-D-Phe1-Tyr3-octreotide kinetics in patients with meningiomas. J Nucl Med. 2005:46:763-9.

30. Lau EW, Drummond KJ, Ware RE, Drummond E, Hogg A, Ryan G, Grigg A, Callahan J, Hicks RJ. Comparative PET study using F-18 FET and F-18 FDG for the evaluation of patients with suspected brain tumour. J Clin Neurosci. 2010;17:43-9.

31. Langen K, Hamacher K, Weckesser M, Floeth F, Stoffels G, Bauer D, Coenen HH, Pauleit D. O-(2-[18F]fluoroethyl)-L-tyrosine: uptake mechanisms and clinical applications. Nucl Med Biol. 2006;33:287-94.

32. Gudjonsson O, Blomquist E, Lilja A, Ericson H, Bergstrom M, Nyberg G. Evaluation of the effect of high-energy proton irradiation treatment on meningiomas by means of 11C-L-methionine PET. Eur J Nucl Med. 2000;27:1793-9.

33. Combs SE, Welzel T, Habermehl D, Rieken S, Dittmar JO, Kessel K, Jakel O, Haberkorn U, Debus J. Prospective evaluation of early treatment outcome in patients with meningiomas treated with particle therapy based on target volume definition with MRI and 68Ga-DOTATOC-PET. Acta Oncol. 2013;52:514-20.

34. Afshar-Oromieh A, Giesel FL, Linhart HG, Haberkorn U, Haufe S, Combs SE, Podlesek D, Eisenhut M, Kratochwil C. Detection of cranial meningiomas: comparison of (6)(8)Ga-DOTATOC PET/CT and contrast-enhanced MRI. Eur J Nucl Med Mol Imaging. 2012;39:1409-15.

\section{Submit your next manuscript to BioMed Central and we will help you at every step:}

- We accept pre-submission inquiries

- Our selector tool helps you to find the most relevant journal

- We provide round the clock customer support

- Convenient online submission

- Thorough peer review

- Inclusion in PubMed and all major indexing services

- Maximum visibility for your research

Submit your manuscript at www.biomedcentral.com/submit 